\title{
Separation of particles by size from a suspension using the motion of a confined bubble
}

Cite as: Appl. Phys. Lett. 112, 181604 (2018); https://doi.org/10.1063/1.5023341

Submitted: 23 January 2018 . Accepted: 16 April 2018. Published Online: 04 May 2018

Yingxian Estella Yu (D), Sepideh Khodaparast, and Howard A. Stone (D)
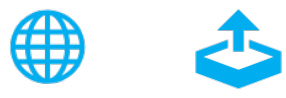

\section{ARTICLES YOU MAY BE INTERESTED IN}

Salt type and concentration affect the viscoelasticity of polyelectrolyte solutions

Applied Physics Letters 112, 203701 (2018); https://doi.org/10.1063/1.5026573

Formation of dispersions using "flow focusing" in microchannels

Applied Physics Letters 82, 364 (2003); https://doi.org/10.1063/1.1537519

Quick deposition of a fluid on the wall of a tube

Physics of Fluids 12, 2367 (2000); https://doi.org/10.1063/1.1289396

\section{Lock-in Amplifiers up to $600 \mathrm{MHz}$}
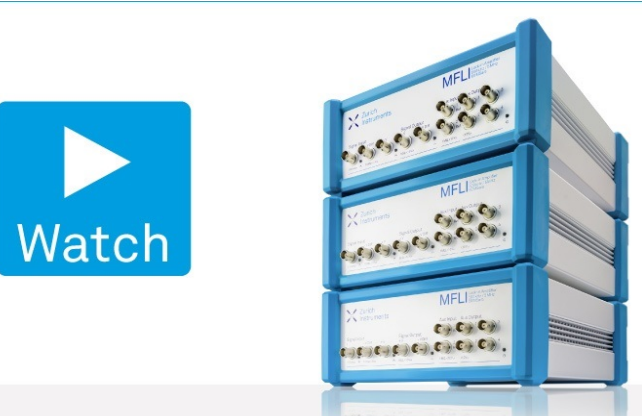


\title{
Separation of particles by size from a suspension using the motion of a confined bubble
}

\author{
Yingxian Estella Yu, ${ }^{1}$ Sepideh Khodaparast, ${ }^{1,2}$ and Howard A. Stone ${ }^{1, a)}$ \\ ${ }^{1}$ Department of Mechanical and Aerospace Engineering, Princeton University, Princeton, New Jersey 08544, \\ USA \\ ${ }^{2}$ Department of Chemical Engineering, Imperial College London, London SW7 2AZ, United Kingdom
}

(Received 23 January 2018; accepted 16 April 2018; published online 4 May 2018)

\begin{abstract}
When confined in a liquid-filled circular cylinder, a long air bubble moves slightly faster than the bulk liquid as a small fraction of the liquid leaks through a very thin annular gap between the bubble and the internal wall of the cylinder. At low velocities, the thickness of this lubricating film formed around the bubble is set only by the liquid properties and the translational speed of the bubble and thus can be tuned in a simple fashion. Here, we use this setting to filter, based on size, micron-size particles that are originally dispersed in a suspension. Furthermore, we apply this process for separation of particles from a polydisperse solution. The bubble interface is free of particles initially, and particles of different sizes can enter the liquid film region. Particle separation occurs when the thickness of the lubricating liquid film falls between the diameters of the two different particles. While large particles will be collected at the bubble surface, smaller particles can leak through the thin film and reach the fluid region behind the bubble. As a result, the film thickness can be fine-tuned by simply adjusting the speed of a translating confined bubble, so as to achieve separation of particles by size based on the relative particle diameter compared to the film thickness. Published by AIP Publishing. https://doi.org/10.1063/1.5023341
\end{abstract}

There is a continuously increasing demand for technologies offering separation of micron- and nano-sized particles from polydisperse solutions for the purpose of filtration or sorting in chemical engineering, medical/pharmaceutical applications, and environmental assessment. Conventional separation techniques involve centrifugation, ${ }^{1,2}$ membrane filtration, ${ }^{3-5}$ and field-flow fractionation, ${ }^{6}$ which often suffer from separation resolution and efficiency reduction due to clogging. Recent studies have focused on membraneless particle separation techniques, which can be grouped into one of the two categories: passive separation and active separation. ${ }^{7}$ While passive separation processes make use of the interactions among particles and the hydrodynamic flow field, ${ }^{8-15}$ active separation processes are studied involving external fields, ${ }^{16-20}$ which aim to improve the separation efficiency. Most of these recent advanced studies have focused on a microfluidics approach that allows higher sorting resolution for smaller sample volumes. ${ }^{21}$ However, such approaches are often system-specific, i.e., the particle separation resolution is highly dependent on the specific channel geometry. Moreover, some external fields may lead to undesirable interactions with the samples, especially for cell sorting applications. In this work, we introduce a size sorting and separation procedure for microparticles, which relies on the interface of a moving bubble confined in small channels. We show that the bubble-driven sorting technique provides a high extraction efficiency and shows a self-healing behavior that prevents clogging.

The translation of a long bubble confined in a circular capillary of radius $R$ filled with pure liquid of viscosity $\mu$ and surface tension $\gamma$ is well documented in the literature, ${ }^{22-29}$ in

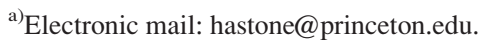

contrast to that in a capillary filled with a complex liquid, such as a colloidal suspension. ${ }^{30-33}$ In a reference frame translating at the bubble velocity $U_{b}$, most of the liquid phase in the front and at the back of the bubble remains on separate streamlines and do not mix with one another. However, a fraction of the liquid ahead of the bubble leaks towards the back of the bubble through an annular liquid-filled gap of uniform thickness $b$ around the bubble [Fig. 1(a)]. In the limit of negligible buoyancy and inertial effects, the normalized thickness

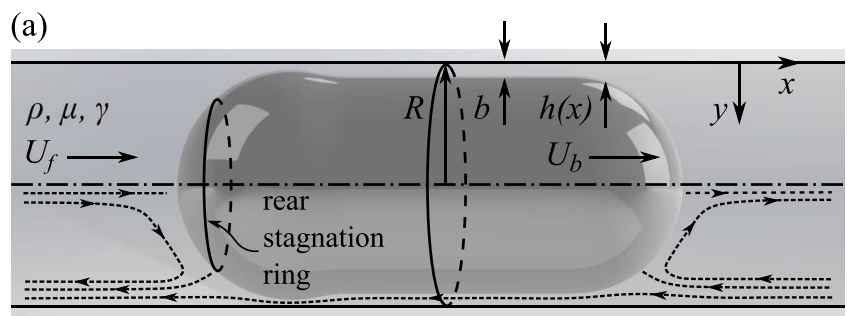

(b)

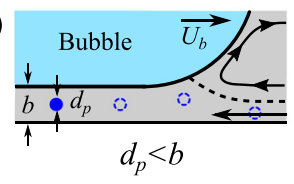

(c)

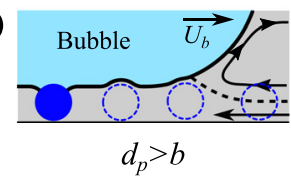

FIG. 1. Schematics of the flow field around a confined bubble. (a) A thin annular gap of thickness $b$ is formed at the wall when a confined bubble translates at the speed $U_{b}$ in a capillary of radius $R$. The lower half of the schematic illustrates the typical streamlines in the liquid phase at $C a, \operatorname{Re}, \mathrm{Bo} \ll 1$ in a reference frame translating with the bubble. (b) and (c) The hypothetical flow configurations in the liquid-filled annular gap at the nose of the bubble translating in a solution of monodisperse colloids. (b) The diameter of the colloids is smaller than the thickness of the gap $d_{p}<b$, and thus, they are expected to move through the thin film region without colliding with the bubble interface. (c) In the case of $d_{p}>b$, colloids may enter the thin film region by deforming the bubble interface and are captured by the interface due to the rupture of the liquid film between the particle and the bubble surface. 
of the pure liquid film is determined by the capillary number ${ }^{26}$ $C a \equiv \mu U_{b} / \gamma$

$$
\frac{b}{R}=\frac{1.34 C a^{2 / 3}}{1+3.5 C a^{2 / 3}} .
$$

In this letter, we show the potential of utilizing this tuneable gap and the flow field around a confined bubble for separation of particles by size in colloidal suspensions.

Following the flow field close to the wall, a fraction of the particles ahead of the bubble are directed backward through the annular gap. We consider a surfactant-free and dilute Newtonian colloidal suspension (CS) with non-polar solute and uncharged particles fully wetted by the liquid. With $h(x)$ the thickness of the fluid gap between the bubble surface and the tube inner wall, particles with diameter $d_{p}$ smaller than $h(x)$ at the front stagnation ring are expected to enter the thin film region. ${ }^{34}$ We may consider that the particle-interface interaction is due to the intermolecular attractions, such as van der Waals forces. Thus, these particles are expected to experience at least two distinct fates, depending on the ratio of their diameter $d_{p}$ to the uniform film thickness $b$, i.e., $d_{p} / b$ [Figs. 1(b) and 1(c)]: (i) if a particle of diameter $d_{p}<b$ enters the thin liquid film, the liquid gap between the free interface and the particle boundary at most $b-d_{p}$. This film may rupture and cause the particle to rest on the interface when the intermolecular forces overcome the capillary effects, which requires the thin liquid gap to be as small as hundreds of nanometers, if not tens of nanometers. Therefore, unless $d_{p} \approx b$, or the particles are sliding in close vicinity to the bubble surface, suspended colloids with diameters smaller than the liquid film thickness $\left(b_{p} / b<1\right)$ are expected to reach the region behind the bubble without interacting significantly with the interface [see Fig. 1(b)]. (ii) However, for particles with diameters sufficiently larger than the thickness of the film $\left(b_{p} / b>1\right)$, the probability of particle absorption on the interface increases significantly [Fig. 1(c)]. Since surface tension counteracts the interface deformation, large particles in the thin film region can be considered as always sliding in close vicinity to the bubble surface. Therefore, we expect the bubble surface to absorb the larger particles entering the thin film region and serve as a filter for colloids of diameters larger than the thickness of the liquid film $\left(b_{p} / b>1\right)$.

To verify the existence of the two distinct fates for colloids based on $d_{p} / b$, we perform experiments in a $L=50 \mathrm{~cm}$ long circular glass capillary with an inner radius of $R=0.56 \mathrm{~mm}$ in a refractive index-matched setup. ${ }^{35}$ The glass capillary is initially filled with a CS of neutrally buoyant poly(methyl methacrylate) (PMMA) particles that serves as the working fluid ahead of the bubble. Next, a flexible tube is connected to a syringe filled with the pure liquid (glycerol), which is the working fluid behind the bubble. A short length at the end of the flexible tubing is left unfilled so that an air plug forms upon connecting the tubing to the glass capillary and starting the syringe pump at the flow-rate of interest. A sequence of brightfield images are captured at the center-plane of the glass capillary as the bubble translates along the channel. More details of the experimental setup and properties of the different CS used in the experiments are listed in the supplementary material.

In the first experiment, we choose $C a=1.34 \times 10^{-2}$ and a CS with particles of diameter $d_{p}=10 \mu \mathrm{m}$ in front of the bubble. Based on the prediction of the film thickness from Eq. (1), this configuration allows testing the condition (i) above, where $d_{p} / b<1$. Figure 2(a) shows that an annular stream of CS leaks through the thin film region and reaches the fluid at the back of the bubble. In contrast, when using a suspension of larger particles $d_{p}=40 \mu \mathrm{m}$ in front of the bubble translating at the same $\mathrm{Ca}$, leading to condition (ii) above, where $d_{p} / b>1$, no particle reaches the liquid behind the bubble [Fig. 2(b)]. The larger particles are, however, found forming a monolayer coating at the back of the bubble. ${ }^{33}$ In order to test the sensitivity of the system to the thickness of the liquid gap, we increase the $\mathrm{Ca}$ downstream in the same experiment to reach the condition $d_{p} / b<1$ again [Fig. 2(c)]. In contrast to Fig. 2(b), the thicker liquid gap at higher $C a$ allows the same $40 \mu \mathrm{m}$ particles to leak towards the back of the bubble, i.e., the gap size and the
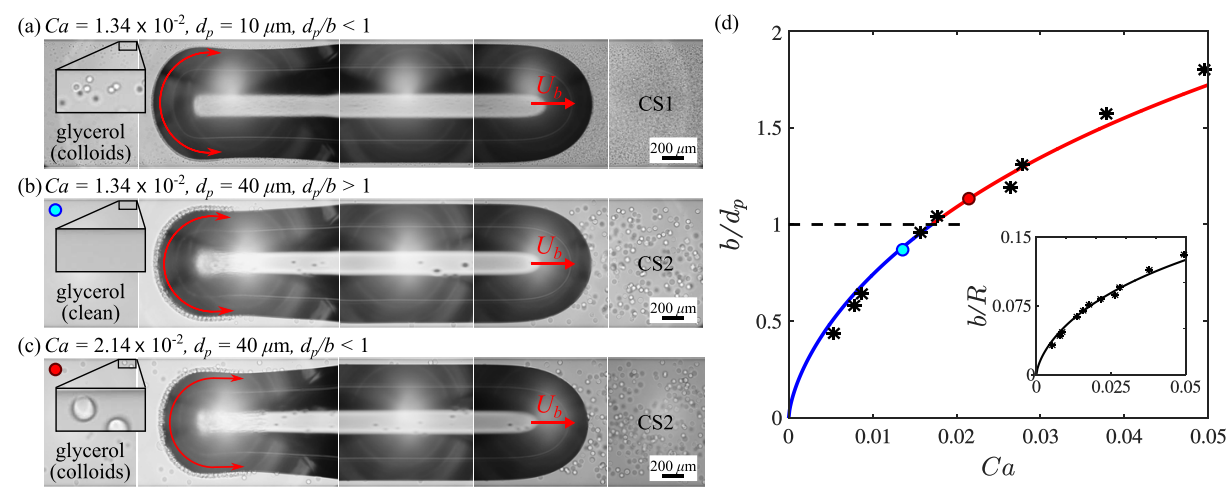

FIG. 2. Particle filtration by tuning the capillary number $C a$ and consequently the liquid film thickness $b$. Monodisperse suspensions CS1 $\left(d_{p}=10 \mu \mathrm{m}\right)$ and CS2 $\left(d_{p}=40 \mu \mathrm{m}\right)$ are used as the working liquids in front of the bubble, while pure glycerol fills the capillary behind the bubble. (a) Bubble translates at $C a=1.34 \times 10^{-2}(b=35 \mu \mathrm{m})$ through CS1. $d_{p} / b<1$ and particles that pass through the annular gap reach the pure glycerol behind the bubble. (b) Bubble translates at $C a=1.34 \times 10^{-2}(b=35 \mu \mathrm{m})$ through CS2. $d_{p} / b>1$ and all the particles are captured by the bubble interface. (c) A bubble translates at a higher speed compared to panel (b), $C a=2.14 \times 10^{-2}(b=46 \mu \mathrm{m})$, through the same CS (CS2); therefore, the condition on the relative particle size is switched to $d_{p} / b<1$ and particles now appear in the fluid behind the bubble. Note that particle coating on the interface is observed in all three cases (a)-(c). (d) The thickness of the uniform liquid film $b$ as a function of $C a$. CS2 is used in this set of experiments. A clear transition between the leakage through the thin film and filtration by the bubble interface is observed at $b / d_{p} \approx 1$. The blue and red closed markers refer to cases in panels (b) and (c), respectively. The inset shows measurements of the normalized liquid film thickness $b / R$ in comparison to the theoretical prediction (black solid line) provided in Eq. (1). 
bubble-driven filtration capacity can be tuned in-situ simply by varying the flow-rate of the liquid phase.

Furthermore, we perform experiments by systematically varying the speed of the bubble and consequently the capillary number in the range $5 \times 10^{-3}<C a<5 \times 10^{-2}$, while keeping the diameter of the particles constant at $d_{p}=40 \mu \mathrm{m}$ [Fig. 2(d)]. The results of these experiments demonstrate the particle-filtering capability of the system without modifying the setup. As presented in [Fig. 2(d)], the threshold for the relative film thickness to allow passage of particles from the front to the back of the bubble is close to $b / d_{p}=1$, as predicted above. Note that coating the bubble by a monolayer of larger particles modifies the boundary condition at the bubble interface, causing an increase in the film thickness in the coated section of the interface, ${ }^{33}$ and thus naturally prevents clogging of the system.

Unlike the experiments performed in previous studies, ${ }^{30-33}$ here we use different working fluids ahead (CS) and behind (pure glycerol) the bubble, as shown in Fig. 2. The pure liquid at the back of the bubble provides a reservoir to collect the separated particles in our system. Furthermore, Fig. 3 shows that, regardless of the ratio $d_{p} / b$, no specific interaction occurs between the bubble interface and the colloids if the CS is placed behind the bubble instead, while the front is pure glycerol. Therefore, the fluid at the back of the bubble does not affect the particle filtration or transport process, which allows a preferential direction in the filtration process. In general, irreversibility is a desirable feature in filtration and/or separation processes, i.e., particles separated from a CS are to be kept away from the original solution at all times. The directional feature shown in Figs. 2 and 3 demonstrates that the current system serves this goal.

We next examine the interaction between the surface of a confined bubble and a bidisperse suspension. We refer to this process as separation here and test the corresponding capacity of the bubble-driven system for a bidisperse CS composed of small and large colloids of diameters $d_{p, s}$ and $d_{p, \ell}$ in glycerol. In each experiment, the capillary number $\mathrm{Ca}$ is adjusted so that the corresponding thickness of the annular gap falls in between the two particle sizes, $d_{p, s}<b<d_{p, \ell}$. The system is initially filled with the bidisperse CS followed by a confined bubble and pure glycerol behind the bubble, which serves as a collection reservoir for the smaller particles with diameter $d_{p, s}$. The particle size distribution is analyzed before and after the particle separation process, from a

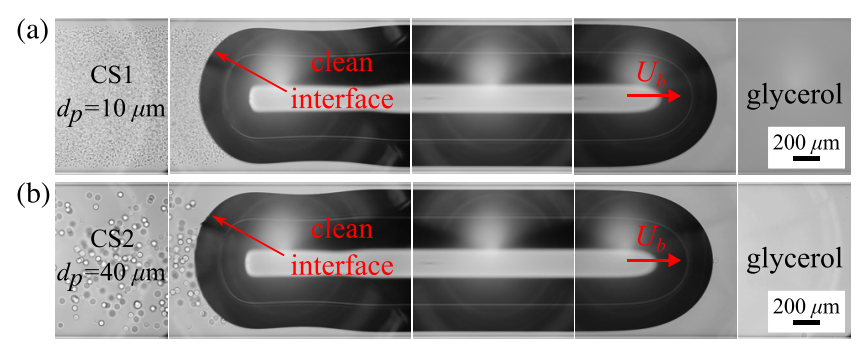

FIG. 3. CS as working fluid behind the bubble and directional separation. Bubbles are translating at $C a=1.34 \times 10^{-2}$, with pure glycerol in front of the bubbles and CS [CS1 for panel (a), where $d_{p} / b<1$, and CS2 for panel (b), where $d_{p} / b>1$ ] behind the bubble. In both cases, no interaction is observed between the particles and the bubble interfaces, which remain free of particles.
$200 \mu \mathrm{L}$ sample of working fluids ahead and behind the bubble, respectively. For more details, refer to the supplementary material.

First, we use a bidisperse suspension CS3 with particles of diameters $d_{p, s}=10 \mu \mathrm{m}$ and $d_{p, \ell}=40 \mu \mathrm{m}$, whose volume fractions are $\phi_{s}=5.1 \times 10^{-3}$ and $\phi_{\ell}=5.1 \times 10^{-2}$, respectively. The volume fraction ratio $\phi_{\ell} / \phi_{s}=10$ is chosen to intensify the separation effect, since the number density ratio would be $n_{s} / n_{\ell}=\left(d_{p, \ell} / d_{p, s}\right)^{3}=64$ if the same volume fractions were used. As the bubble translates through CS3 at $C a=8.65 \times 10^{-3}$, a clean uniform film of thickness $b=26.0 \mu \mathrm{m}$ is formed at the wall, see Fig. 4(a). Initially, both large and small particles enter the thin film region. While all larger particles are captured and collected at the rear section of the bubble interface, the majority of the smaller particles originally in the thin film region leak to the fluid behind the bubble. As a result, the smaller particles are separated from the bidisperse suspension. A histogram comparing the particle number densities before and after the experiment is shown in Fig. 4(b), with the results before and after the experiment shown in red and blue, respectively. Compared to the particle size distribution before the experiment, the peaks for large particles are eliminated after the bubble passes by, with the separation rate for small particles being $100 \%$ for this specific experiment.

A second experiment is performed with a more challenging sample, a bidisperse suspension CS4 with $d_{p, s}=10 \mu \mathrm{m}$ and $d_{p, \ell}=20 \mu \mathrm{m}$, whose volume fractions are $\phi_{s}=\phi_{\ell}=5.3$ $\times 10^{-3}$. The volume fraction is chosen to be the same in this case, since the particle size difference is much smaller in this suspension. The bubble translates through CS4 at $\mathrm{Ca}=4.24$ $\times 10^{-3}$, leaving a uniform thin film of thickness $b=17.0 \mu \mathrm{m}$. As shown in Fig. 4(c), both large and small particles enter the thin film region. The footprints shown in the image are much smaller due to the small difference between $b$ and $d_{p, \ell}$ in this case. Particle size distributions before and after the experiment are displayed in Fig. 4(d), showing a much reduced peak for the large particles. A close examination shows that the number density ratio $n_{s} / n_{\ell}$ in the samples increases from 3.98 (before the experiment) to 34.5 (after the experiment), which indicates a significant reduction of large particles after the separation process.

The current separation method makes use of the relative size between the particle diameter $d_{p}$ and the uniform film thickness $b$. Therefore, we expect that the range of particle sizes that can be separated relates to the limits of the liquid film thickness, which are estimated to be $\mathcal{O}(0.1)$ to $\mathcal{O}(100) \mu \mathrm{m}$; see supplementary material for the details of the estimate. We note that when the bubble interface is completely coated with particles, the thickness of the uniform film will increase up to $2^{2 / 3}$ times of the original value, ${ }^{33}$ after which large particles may also leak to the back of the bubble. However, this is also a beneficial self-healing feature of the system in terms of preventing clogging at all times. Additionally, particle monolayer buckling can occur at the rear stagnation ring if the monolayer area exceeds a critical value. The buckling feature is caused by a critical compressive interfacial shear, over which the rigid particles fail to rearrange, ${ }^{36}$ where little non-Newtonian response should be expected. Therefore, there is a maximum length of a bubble that can prevent buckling from occurring, which 

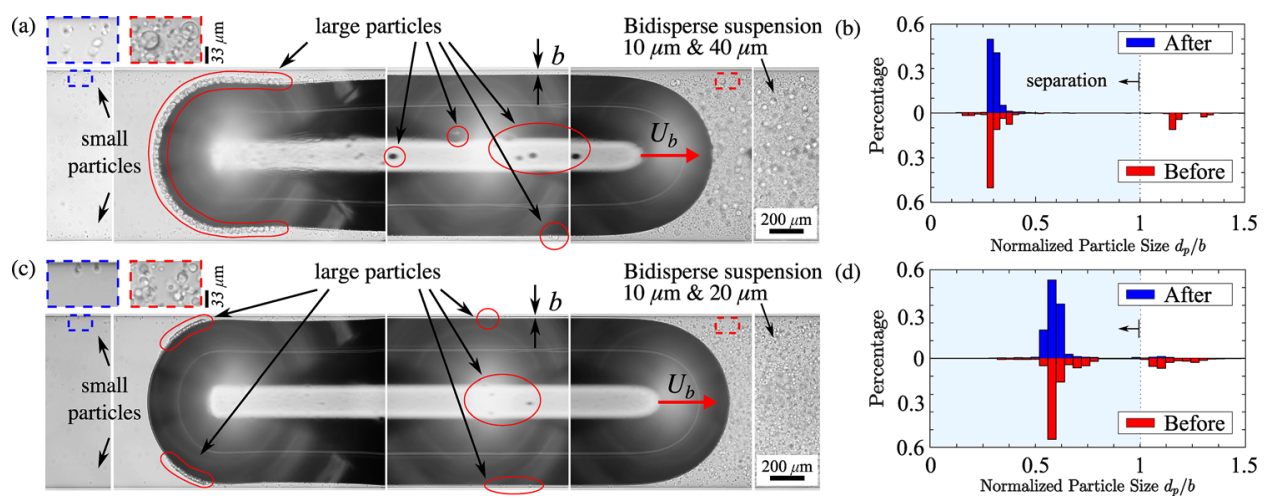

FIG. 4. Separation of particles in bidisperse suspensions. (a) The bubble translates at $C a=8.65 \times 10^{-3}$ (film thickness $b=26.0 \mu \mathrm{m}$ ) into a bidisperse suspension of particles with diameters of $d_{p, s}=10 \mu \mathrm{m}$ and $d_{p, \ell}=40 \mu \mathrm{m}\left(\phi_{s}=5.1 \times 10^{-3}\right.$ and $\left.\phi_{\ell}=5.1 \times 10^{-2}\right)$. Both large and small particles may enter the liquidfilled gap. Among these, all large particles are captured at the bubble interface, while the small particles are transferred to the pure glycerol at the back of the bubble. (b) Particle size distributions in the fluid ahead (red) and behind (blue) the bubble. No trace of the larger particles is found behind the bubble. (c) The bubble translates at $C a=4.24 \times 10^{-3}$ (film thickness $b=17.0 \mu \mathrm{m}$ ) into a bidisperse suspension of particles with diameters of $d_{p, s}=10 \mu \mathrm{m}$ and $d_{p, \ell}=20 \mu \mathrm{m}$ $\left(\phi_{s}=\phi_{\ell}=5.3 \times 10^{-3}\right)$. (d) Particle size distributions in the liquid ahead (red) and behind (blue) the bubble. The number density ratio $n_{s} / n_{\ell}$ in the sample increases from 3.98 (before the experiment) to 34.5 (after the experiment).

ensures a desirable separation process. But both limitations can be overcome by inserting new bubbles into the tube, to ensure continuous success of the separation process.

The present particle separation process utilizes a millimeter scale capillary tube and thus provides a chance for scaling up the particle separation process. This process also provides a flexible way to separate different polydisperse particle batches in the same experimental setup, since the critical dimension - the thickness of the uniform fluid film around the bubble-is only determined by the capillary number $\mathrm{Ca}$, which can be well-controlled by solely manipulating the flow-rate of the incoming flow. The results shown here provide a cost-effective particle separation technique. Moreover, the present methodology can also be useful for sorting cells since it has a minimal impact on the sample.

See supplementary material for additional material properties and experimental details.

Y.E.Y. and H.A.S. are grateful for partial support from the NSF Training Grant in the Mathematical Methods for Water Problems (Grant No. AWD1004562, PI $=$ P. Constantin). Y.E.Y. thanks the Eli and Britt Harari University Fellowship Fund for partial support. H.A.S. thanks M. Bazant (MIT) and O. H. Kim (Saint-Gobain Research) for helpful conversations.

${ }^{1}$ P. E. Lindahl, "Principle of a counter-streaming centrifuge for the separation of particles of different sizes," Nature 161, 648-649 (1948).

${ }^{2}$ C. A. Schlutz, "Centrifuge separation and washing device and method," U.S. patent 3,982,691 (1976).

${ }^{3}$ R. W. Sheldon, "Size separation of marine seston by membrane and glassfiber filters," Limnol. Oceanogr. 17, 494-498 (1972).

${ }^{4}$ R. W. Baker, Membrane Technology (Wiley Online Library, 2000).

${ }^{5} \mathrm{D}$. Aussawasathien, C. Teerawattananon, and A. Vongachariya, "Separation of micron to sub-micron particles from water: Electrospun nylon-6 nanofibrous membranes as pre-filters," J. Membr. Sci. 315, 11-19 (2008).

${ }^{6}$ J. C. Giddings, "Field-flow fractionation: Analysis of macromolecular, colloidal, and particulate materials," Science 260, 1456-1466 (1993).

${ }^{7}$ C. W. Shields IV, C. D. Reyes, and G. P. López, "Microfluidic cell sorting: A review of the advances in the separation of cells from debulking to rare cell isolation," Lab Chip 15, 1230-1249 (2015).
${ }^{8}$ J. Oakey, J. Allely, and D. W. M. Marr, "Laminar-flow-based separations at the microscale," Biotechnol. Prog. 18, 1439-1442 (2002).

${ }^{9}$ M. Yamada, M. Nakashima, and M. Seki, "Pinched flow fractionation: Continuous size separation of particles utilizing a laminar flow profile in a pinched microchannel," Anal. Chem. 76, 5465-5471 (2004).

${ }^{10}$ A. A. S. Bhagat, S. S. Kuntaegowdanahalli, and I. Papautsky, "Continuous particle separation in spiral microchannels using dean flows and differential migration," Lab Chip 8, 1906-1914 (2008).

${ }^{11}$ J.-S. Park and H.-I. Jung, "Multiorifice flow fractionation: Continuous size-based separation of microspheres using a series of contraction/expansion microchannels," Anal. Chem. 81, 8280-8288 (2009).

${ }^{12}$ S. S. Kuntaegowdanahalli, A. A. S. Bhagat, G. Kumar, and I. Papautsky, "Inertial microfluidics for continuous particle separation in spiral microchannels," Lab Chip 9, 2973-2980 (2009).

${ }^{13}$ L. R. Huang, E. C. Cox, R. H. Austin, and J. C. Sturm, "Continuous particle separation through deterministic lateral displacement," Science 304, 987-990 (2004).

${ }^{14}$ S.-C. Kim, B. H. Wunsch, H. Hu, J. T. Smith, R. H. Austin, and G. Stolovitzky, "Broken flow symmetry explains the dynamics of small particles in deterministic lateral displacement arrays," Proc. Natl. Acad. Sci. U. S. A. 114, E5034-E5041 (2017).

${ }^{15}$ S. Shin, O. Shardt, P. B. Warren, and H. A. Stone, "Membraneless water filtration using $\mathrm{CO}_{2}$," Nat. Commun. 8, 15181 (2017).

${ }^{16} \mathrm{D}$. Holmes and H. Morgan, "Cell positioning and sorting using dielectrophoresis," Eur. Cells Mater. 4, 120-122 (2002).

${ }^{17}$ N. Pamme, J. C. T. Eijkel, and A. Manz, "On-chip free-flow magnetophoresis: Separation and detection of mixtures of magnetic particles in continuous flow," J. Magn. Magn. Mater. 307, 237-244 (2006).

${ }^{18}$ D. McGloin, "Optical tweezers: 20 years on," Philos. Trans. R. Soc. A 364, 3521-3537 (2006).

${ }^{19}$ S. F. Ibrahim and G. van den Engh, "Flow cytometry and cell sorting," in Cell Separation (Springer, 2007), pp. 19-39.

${ }^{20} \mathrm{X}$. Lin, J. Yao, H. Dong, and X. Cao, "Effective cell and particle sorting and separation in screen-printed continuous-flow microfluidic devices with 3D sidewall electrodes," Ind. Eng. Chem. Res. 55, 13085-13093 (2016).

${ }^{21}$ D. R. Gossett, W. M. Weaver, A. J. Mach, S. C. Hur, H. T. K. Tse, W. Lee, H. Amini, and D. Di Carlo, "Label-free cell separation and sorting in microfluidic systems," Anal. Bioanal. Chem. 397, 3249-3267 (2010).

${ }^{22}$ F. Fairbrother and A. E. Stubbs, "119. Studies in electro-endosmosis. Part VI. The 'bubble-tube' method of measurement," J. Chem. Soc. 0, 527-529 (1935).

${ }^{23}$ F. P. Bretherton, "The motion of long bubbles in tubes," J. Fluid Mech. 10, 166-188 (1961).

${ }^{24}$ G. I. Taylor, "Deposition of a viscous fluid on the wall of a tube," J. Fluid Mech. 10, 161-165 (1961).

${ }^{25} \mathrm{H}$. Wong, C. J. Radke, and S. Morris, "The motion of long bubbles in polygonal capillaries. Part 1. Thin films," J. Fluid Mech. 292, 71-94 (1995).

${ }^{26} \mathrm{P}$. Aussillous and D. Quéré, "Quick deposition of a fluid on the wall of a tube," Phys. Fluids 12, 2367-2371 (2000).

${ }^{27}$ M. Heil, "Finite Reynolds number effects in the Bretherton problem," Phys. Fluids 13, 2517-2521 (2001). 
${ }^{28}$ S. Khodaparast, M. Magnini, N. Borhani, and J. R. Thome, "Dynamics of isolated confined air bubbles in liquid flows through circular microchannels: An experimental and numerical study," Microfluid. Nanofluid. 19, 209-234 (2015).

${ }^{29}$ O. Atasi, S. Khodaparast, B. Scheid, and H. A. Stone, "Effect of buoyancy on the motion of long bubbles in horizontal tubes," Phys. Rev. Fluids 2, 094304 (2017).

${ }^{30}$ A. B. Subramaniam, J. Wan, A. Gopinath, and H. A. Stone, "Semi-permeable vesicles composed of natural clay," Soft Matter 7, 2600-2612 (2011).

${ }^{31}$ A. P. Kotula and S. L. Anna, "Probing timescales for colloidal particle adsorption using slug bubbles in rectangular microchannels," Soft Matter 8, 10759-10772 (2012).
${ }^{32}$ F. Zoueshtiagh, M. Baudoin, and D. Guerrin, "Capillary tube wetting induced by particles: Towards armoured bubbles tailoring," Soft Matter 10, 9403-9412 (2014).

${ }^{33}$ Y. E. Yu, S. Khodaparast, and H. A. Stone, "Armoring confined bubbles in the flow of colloidal suspensions," Soft Matter 13, 2857-2865 (2017).

${ }^{34}$ C. E. Colosqui, J. F. Morris, and H. A. Stone, "Hydrodynamically driven colloidal assembly in dip coating,” Phys. Rev. Lett. 110, 188302 (2013).

${ }^{35}$ S. Khodaparast, N. Borhani, and J. R. Thome, "Application of micro particle shadow velocimetry $\mu$ PSV to two-phase flows in microchannels," Int. J. Multiphase Flow 62, 123-133 (2014).

${ }^{36}$ D. Vella, P. Aussillous, and L. Mahadevan, "Elasticity of an interfacial particle raft," Europhys. Lett. 68, 212 (2004). 